\title{
New Soluble Pentacene Precursors for the Application of Organic Thin-Film Transistors
}

\author{
Meyoung Ju Joung, Jin Hee Ahn, 'Seung Youl Kang, Kyu Ha Baek, Seong Deok Ahn, \\ Lee Mi Do, Chul Am Kim, Gi Heon Kim, In Kyu You, Sung Min Yoon, and Kyung Soo Suh* \\ Applied Devices Deparment, Electronics and Telecommunications Research Institute, \\ 161 Gajeong-Dong. Yusung-Gu. Daejeon 305-350. Korea \\ Medicinal Science Division, Korea Research Institute of Chemical Technologv. \\ 100 Jang-Dong, Yusung-Gu, Daejeon 305-600, Korea \\ Recerved September 16, 2003
}

Key Words : Soluble pentacene precursor. Organic thin film transistor. Diels-Alder reaction. Organic semiconductor

Oter the past two decades, organic thin-film transistors (OTFTs) based on organic semiconductors have been envisioned as a viable altenative to more traditional TFTs based on inorganic material, such as $\mathrm{Si}$ and $\mathrm{Ge}$, which have charge carrier mobilities about three orders of magnitude higher. The OTFTs have been used for switching devices of low-cost electronic application such as integrated circuits. ${ }^{1}$ chemical vapor sensors ${ }^{2}$ and active-matrix flat-panel displays (AMFPDs) for examples. liquid crystal pixels (AMLCDs) ${ }^{3}$ or organic light-emitting diodes (AMOLEDs). ${ }^{4}$ Research into organic semiconductors often focuses either on solution processable semiconducting polymer or on snall molecules. Solution processed semiconducting materials are of particular interest for large area coverage. structural flexibility. low-temperature processing, and. especially. ultralow-cost application since they allow the use of spin-coating. spraycoating. screen printing. or ink-jet printing.

As seniconducting polymer for OTFTs. poly (3-hexylthiophene) (P3HT) was fabricated by solution process, the devices showed field effect mobilities ranging from 0.05 to $0.1 \mathrm{~cm}^{2} / \mathrm{Vs}$ and on-off current ratios of $>10^{6}$. Another active layer polymer is poly(9.9-dioctylfluorene-co-bithiophene) (F8T2). ${ }^{6}$ which was spin-coated from sylene solution and high mobility of $0.02 \mathrm{~cm}^{2} / \mathrm{Vs}$ and on-off current ratios of $10^{2}$ were obtained. The high mobility $0.22 \mathrm{~cm}^{2} / \mathrm{Vs}$ has been reported for OTFTs using precursor-route poly (2,5-thienylene) (PTV) ${ }^{7}$ as semiconducting polymer.

Snall molecule semiconductors, such as oligothiophenes and the hydrocarbons (pentacene and tetracene). may be suitable for applications requiring electrical performance similar to that of amorphous silicon TFTs. Among them. pentacene is the most promising organic semiconductor since it offers higher mobility. better on-off ratio, intproved envirommental stability. and better reliability then most other organic seniconductors. ${ }^{8}$ It has been reported that the OTFTs using vacuum-deposition pentacene gave the mobilities of $1 \mathrm{~cm}^{2} / \mathrm{Vs}{ }^{9}$ The main drawback of pentacene for the application of solution process is that it is essentially insoluble in organic solvent at room temperature

Over the last two years. several attempts have been made to synthesize soluble pentacene derivatives or pentacene precursors. One of the potentially promissing pentacene derivatives, 6.13-disubstituted pentacene derivatives have been reported. ${ }^{11}$ Another approaches for the soluble pentacene have been attempted using retro Diels-Alder reactions. Mullen and co-workers ${ }^{11}$ reported the preparation of a soluble precursor of pentacene that was spin-coated from methylene chloride and then heated at $200^{\circ} \mathrm{C}$ to form pentacene via retro Diels-Alder reaction. The OTFTs with above precursor showed field-effect mobilities of 0.1-0.2 $\mathrm{cm}^{2} / \mathrm{Vs}$, however the conversion temperature is too high for plastic substrate application and its prepared in muti-step reactions with low overall yield. Recently. researchers at $\mathrm{IBM}^{12}$ synthesized more reactive pentacene precursor via hetero Diels-Alder reaction with reactive dienophile. $\mathrm{N}$ sulfinylacetanide. After this precursor was spin-coated then heated at ranging $120-200^{\circ} \mathrm{C}$ to give pentacene via retro Diels-Alder reaction. Mobilities as high as ranging from 0.13 to $0.89 \mathrm{~cm}^{2} / \mathrm{Vs}$ and on-off ratio of above $2 \times 10^{7}$ were achieved. However the preparation and handling of very unstable dienophile. $\mathrm{N}$-sulfinylacetamide. is troublesome. Therefore more simple and easier synthetic method for the reactive pentacene precursor. which can be easily decomposed at low temperature, is demanded for solution processible OTFTs.

Now we report here the simple method for the preparation of the soluble pentacene precursors, which are the first aza pentacene precursors applicable for the soluble semiconductors. These new candidates can be synthesized from the reaction of commercially available diazo compounds with pentacene in one step. These azodienophiles have been reported that they were reactive in hetero Diels-Alder reactions with acene compounds. ${ }^{12}$ We believe these precursors can be easily converted to pentacene at moderate temperature by retro Diels-Alder reactions that reveal comparable properties to vacuum-deposited pentacene.

Cortesponding author. E-nail: kssullàetri.re.kr 


\section{Results and Discussion}

As shown in Scheme 1, three different kinds of commercially available dialkyl azodicarboxylates reacted with pentacene in chloroform or toluene. These Diels-Alder reactions proceeded without any catalyst under reflux conditions to give Diels-Alder adduct in quantitative to $90 \%$ yields. Excess amount (5 equiv.-10 equiv.) of azo compounds were need for quantitative reactions, the dark blue pentacene was slowly melted reacting with dienophile, then turned to yellow solution after overnight. After evaporation of excess solvent and dienophile under vacuo. the residue was column chromatographed on silica gel (eluent : hexane/EtOAc, $4: 1$ ) to afford white or yellowish solids. These microcrystalline compounds are highly soluble in almost organic solvents and very stable in the air, they can be stored in the refrigerator for six month without any decomposition.
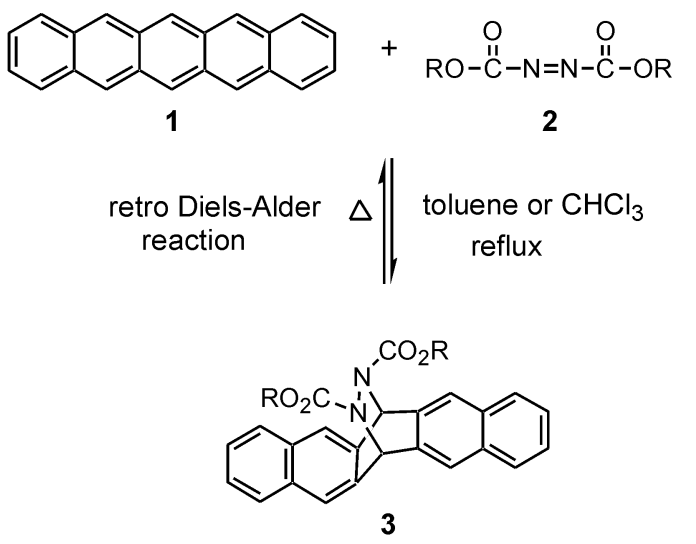

$\mathrm{R}: \mathbf{a}=\mathrm{Me}, \mathbf{b}=\mathrm{Et}$ and $\mathbf{c}=t-\mathrm{Bu}$

The reactivities of the Diels-Alder reactions were changed depending on the $\mathrm{R}$ group of dienophile 2 . In the case of methyl or ethyl group, the azo dienophiles coupled with pentacene in refluxed toluene in quantitative yields. However the reaction of di-tert-butyl azodicarboxylate with pentacene was a little slow and gave better yield of $90 \%$ in

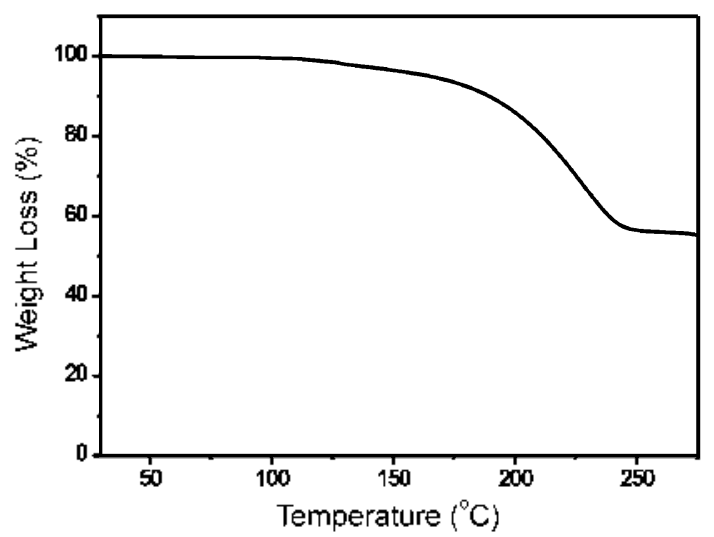

Figure 1. Thermograximetric analysis graph of azo pentacenc precursor (3b) (heating rate: $10^{\circ} \mathrm{C} / \mathrm{min}$ ).

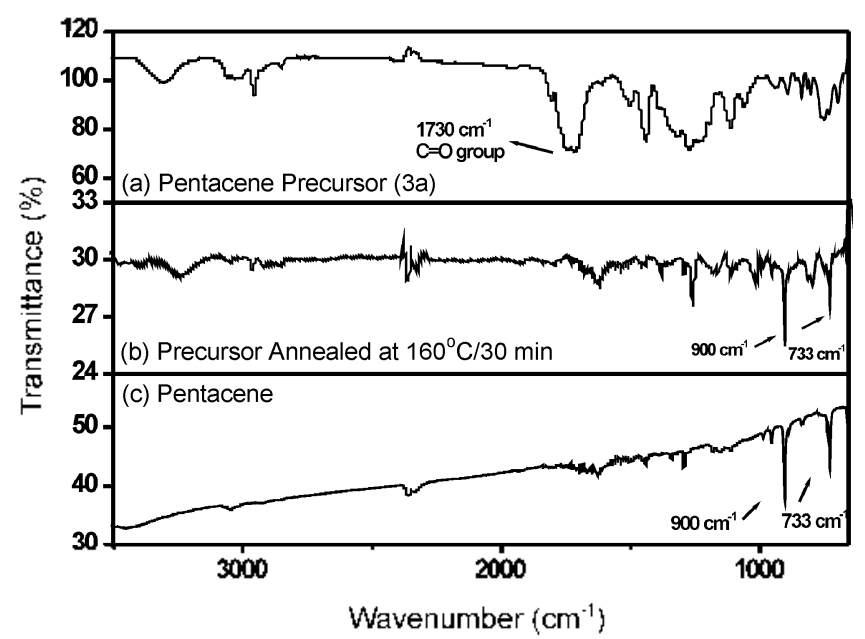

Figure 2. FT-lR spectrums of (a) pentacene precursor (3a. $\mathrm{R}=\mathrm{Me}$ ). (b) pentacene gencrated by annealing of $3 \mathrm{a}$ at $160^{\circ} \mathrm{C}$ for 30 min. and (c) commercially available pertacene.

chloroform than in toluene (Scheme 1). The pentacene precursors (3) were characterized by 'H NMR, F"I-IR. GCMS spectrums, and elemental analysis.

The thermogravimetric analysis graph of pentacene precursor ( $\mathbf{3 b} \mathbf{b}, \mathrm{R}=\mathrm{Et}$ ) is presented in Figure 1 and shows that it decomposes from $170^{\circ} \mathrm{C}$ via retro Diels-Alder reaction. The weight loss of $40 \%$ is accordant to the theoretical weight loss of $38.5 \%$ by the elimination of azo moiety from the precursor $3 \mathbf{b}$. The conversion into pentacene occurs on simple thermal treatment at moderate temperature. The graph could be one of the proofs for the thermal conversion of precursor $3 \mathrm{~b}(\mathrm{R}=\mathrm{Et})$ to pentacene.

Another evidences for the conversion of pentacene precursors to pentacene were detected in $\mathrm{F}[-] \mathrm{R}$ and $\mathrm{GC}$-mass spectrums. Figure 2(b) shows the F"]-IR spectrum of a film of $3 \mathrm{a}(\mathrm{R}=\mathrm{Me})$ thermolyzed for $30 \mathrm{~min}$ at $160^{\circ} \mathrm{C}$ under nitrogen. The characteristic peak of $\mathrm{C}=\mathrm{O}$ stretching band from the starting material $3 a$ at $1730 \mathrm{~cm}^{-1}$ (Fig. 2(a)) is disappeared and two characteristic peaks from pentacene

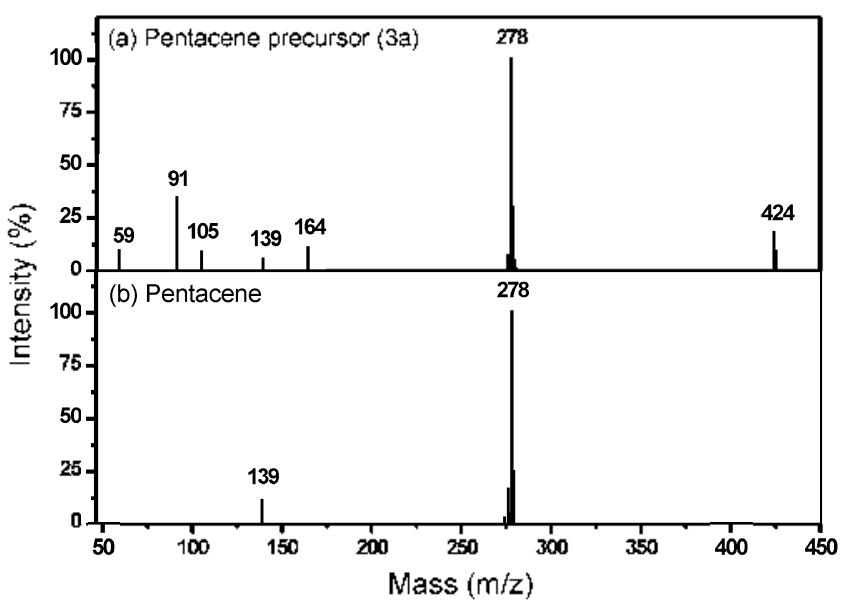

Figure 3. (a) (GC-mass spectrum of the pentacenc precursor (3a). (b) L ibrary mass spectrum of pentacene. 
were observed at $733 \mathrm{~cm}^{-1}$ and $900 \mathrm{~cm}^{-1}$ (Fig. 2(b)). that matches the FT-IR spectrum of pentacene (Fig. 2(c)).

As shown in Figure $3 \mathrm{a}$, a base peak of the pentacene precursor 3a $(\mathrm{R}=\mathrm{Me})$ is detected at $278 \mathrm{~m} / \mathrm{z}$, which is in good agreement with the molecular weight of pentacene. and the patterns of fragmentation of $3 a$ is similar to that of pentacene (Fig. 3b). Those spectrum data suggest that the pentacene precursors can be easily decomposed to pentacene by thermolysis.

Therefore these azo pentacene compounds could be suitable precursors for the generation of pentacene in the solid state and applicable for OTFTs requiring large area coverage. structural flexibility, low-temperature processing. and. especially. low-cost electric applications since they allow the use of spin-coating, spray-coating, screen printing. or ink-jet printing.

We are investigating the fabrication of solution-processed OTFTs with azo pentacene precursors and will publish the applications in the near future. and also we are researching the preparation method of new reactive pentacene precursors. which can make a conversion at very low temperature for the application on plastic substrate.

\section{Experimental Section}

General Procedure of Diels-Alder Reaction. To a slurry of pentacene ( $\mathrm{l} \mathrm{mmol})$ in chloroform or toluene were added azo dienophiles $(5 \cdot 10 \mathrm{mmol}, 5$ equiv -10 equiv) with stirring. The dark blue pentacene was slowly melted reacting with dienophile under reflux, then turned to yellow solution. After overnight. the reaction mixture was evaporated to remove solvent under reduced pressure, then the residue was column chromatographed on silica gel (eluent : hexane/ EtOAc. $4:$ : 1) to afford white or yellowish solids in $90 \%-95$ $\%$ yields.

3a: ${ }^{1} \mathrm{H} N M R\left(\mathrm{CDCl}_{3} .200 \mathrm{MHz}\right) . \delta 3.75$ (s, $\left.3 \mathrm{H}\right), 3.90$ (s. 3 H). 6.53 (s, $2 \mathrm{H}) .7 .30-7.33$ (n. $2 \mathrm{H}), 7.46-7.51(\mathrm{~m}, 4 \mathrm{H})$. 7.80-7.84 (m. 4 H), 7.94 (s, $2 \mathrm{H})$ : FT-IR (neat). $v_{\mathrm{c}=0} 1720 \mathrm{~cm}^{-1}$, $v_{\text {pentacene }} 896 \mathrm{~cm}^{-1}$. $v_{\text {pentacenc }} 754 \mathrm{~cm}^{-1}$ : GCMS m/z (relative intensity) (EI. $70 \mathrm{eV}) 424$ ( $\mathrm{M}^{-}$2). 278 (100. pentacene peak). 3b: ${ }^{1} \mathrm{H}$ NMR $\left(\mathrm{CDCl}_{2}, 200 \mathrm{MHz}\right) . \delta 1.17-1.30(\mathrm{~m}, 6 \mathrm{H})$, $4.11-4.20(\mathrm{~m}, 4 \mathrm{H}) .6 .53$ (s. $2 \mathrm{H}) .7 .45-7.50(\mathrm{~m} .4 \mathrm{H}) .7 .78-$ $7.84(\mathrm{~m}, 6 \mathrm{H}) .7 .94$ (s. $2 \mathrm{H}$ ); GCMS m/z (relative intensity) (EI. $70 \mathrm{eV}) 452\left(\mathrm{M}^{+} 3\right), 278$ (100. pentacene peak).

3c: ${ }^{1} \mathrm{H}$ NMR $\left(\mathrm{CDCl}_{3}, 200 \mathrm{MHz}\right), \delta 1.52(\mathrm{~s}, 9 \mathrm{H}) .1 .48(\mathrm{~s} .9$ H), 6.45 (s. $2 \mathrm{H}), 7.43-7.48(\mathrm{~m}, 4 \mathrm{H}) .7 .76-7.82$ (m. $6 \mathrm{H})$, $7.94(\mathrm{~s}, 2 \mathrm{H})$. Calculated for $\mathrm{C}_{32} \mathrm{H}_{3} \mathrm{~N}_{2} \mathrm{O}_{4}: \mathrm{C} .75 .57 ; \mathrm{H}, 6.34$ : N. 5.51. Found: C. $75.51 ;$ H. 6.35: N. 5.53.

Acknowledgment. We are grateful to the Korea Ministry of Information and Communications for financial support.

\section{References and Notes}

1. Gelinck. G. H.: Geuts. T. C. T.: De Leeuw. D. W. Appl. Phus. Lett. 2000. 77.1487

2. Crone. B:- Dodabalapur, A.: Sarpeshkar, R: Gelperin, A.: Katz. H. E.: Bao, Z. J. Appl Pho 2002, 91, 10140.

3. Schleupen. K.: Alt, P. at al. "High Information Content Color 16.3" Desktop AMLCD with 15.7 Million a-Si:H TFTs". Pwceedings of the $18^{\text {th }}$ International Display Research Confenence. Asta Display '98: 1998: pp 187-190.

4. Riess. W: Riel. H.; Beierlein. T.: Brütting. W.: Müller, P: Seidler. P. F. IBU H J Res \& Dew: $2001,+5.77$.

5. Sirringhaus, H.: Tessler, N.: Friend. R. H. Scince 1998, 280. 1741 .

6. (a) Sirringhaus. H.: Kawase. T.: Friend. R. H.: Shimoda. T.: Inbasekaran. M.: Wu. W.: Woo. E. P. Science 2000. 290.2123, (b) Sirringhaus, H.; Wilson. R. J; Friend. R. H.: Inbasekaran, M.; Wu, W. Woo. E. P.: Grell, M.: Bradlev: D. D. C. Appl. Pho Lett. 2000 77, 406. (c) Shimoda. T. "Ink-jet Technology for Fabrication Processes of Flat Panel Displays". Tech Digest of SID03: $2003: \mathrm{pp}$ $1178-1181$

7. Fuchigami. H.: Tsumura. A.: Koezuka. H. Appl. Phys. Lett. 1993. 63. 1372 .

8. Schon. J. H.: Kloc. Ch.; Batlogg, B. Org. Electron. 2000. I, 57.

9. Schön. J. H. Appl. Phus. Lett. 2001. 79. 4163.

10. (a) Anthony. T. E.: Brooks. J. S.: Eaton. D. L.: Parkin. S. R. J. Am Chem. Soc. 2001. 123. 9482 . (b) Anthony. J. E.: Eaton. D. L.: Parkin. S. R. Org. Lett. 2002. t. 15.

11. Herwig. P. T.: Müllen. K. Adh Mater. 1999, 11.480.

12. Afzali. A.: Dimitrakopoulos, C. D.; Breen, T. L. J. Am. Chent. Soc. $2002,12+, 8812$.

13. Mataka. S.: Ma. T.: Thies. T:: Rudziňski. J. M: Sawada, T: Tashiro. M. Terahedron Lett 1995.36 .6105 